\title{
A new species of Buthus Leach, I8I 5 from Cyprus (Scorpiones, Buthidae)
}

\author{
Ersen Aydın Yağmur ${ }^{1, \dagger}$, Halil Koç2,‡, Wilson R. Lourenço $0^{3, \S}$ \\ I Ege University, Science Faculty, Biology Department, Zoology Section, İzmir, Turkey 2 Sinop University, \\ Science and Art Faculty, Biology Department, Sinop, Turkey 3 Muséum national d'Histoire naturelle, Départe- \\ ment Systématique et Evolution, UMR7205, CP 053, 57 rue Cuvier 75005 Paris, France \\ † urn:lsid:zoobank.org:author:8DB0B243-5B2F-4428-B457-035A8274500C \\ † urn:lsid:zoobank.org:author:77C76C8B-3F8F-4617-8A97-1E55C9F366F7 \\ § urn:lsid:zoobank.org:author:58448BD6-79D7-46CE-AFDD-91EFF2B7D4EF \\ Corresponding author: Ersen Aydın Yağmur (ersen.yagmur@gmail.com) \\ Guest editor: Victor Fet | Received 16 February 2011 | Accepted 25 May 2011 | Published 5 July 2011 \\ urn:lsid:zoobank.org:pub:95309DE0-F756-4756-ABA8-D180A3A42213 \\ Citation: Yağmur EA, Koç H, Lourenço WR (2011) A new species of Buthus Leach, 1815 from Cyprus (Scorpiones, \\ Buthidae). ZooKeys 115: 27-38. doi: 10.3897/zookeys.115.1135
}

\begin{abstract}
During the last decade, several contributions to the genus Buthus Leach, 1815 (family Buthidae) and especially to the 'Buthus occitanus' species complex were proposed. These contributions led to the definition of several species, previously considered only as subspecies or varieties, and also to the description of new species. In the present study, the questionable presence of the genus Buthus in the Cyprus is rediscussed and a new species Buthus kunti sp. n. is described.
\end{abstract}

\section{Keywords}

Scorpion, Buthus, new species, Cyprus

\section{Introduction}

The genus Buthus was described by Leach, 1815 with the type species (by original designation), Scorpio occitanus Amoreux, 1789. The type species was described from Sauvignargues in the South of France. In his study about the scorpions of North Africa,

Copyright E.A.Yağmur et al. This is an open access article distributed under the terms of the Creative Commons Attribution License, which permits unrestricted use, distribution, and reproduction in any medium, provided the original author and source are credited. 
Vachon (see 1952), revised the composition of the genus Buthus and proposed a revised diagnosis, closer to the generic type $B$. occitanus. Consequently, quite many species placed in the genus Buthus have been transferred to other genera. Some were already available as subgenera, while others have been described by Vachon at this occasion. Can be cited, Androctonus Ehrenberg, 1828, Buthacus Birula, 1908, Leiurus Ehrenberg, 1828, Compsobuthus Vachon, 1949 and Buthotus Vachon, 1949 (= Hottentotta Birula, 1908). (see Lourenço 2002, 2003 for details). However, the classification proposed by Vachon (1952) for the species of Buthus, and in particular for those belonging to the "Buthus occitanus" species complex, remained unsatisfactory. A more precise definition of the $B u$ thus species has been attempted recently by Lourenço $(2002,2003)$ which was followed by the elevation of several subspecies to species rank and the description of a new species.

Buthus occitanus (Amoreux, 1789) was first recorded from Cyprus by Kraepelin (1891). Levy and Amitai (1980) confirmed it to Cyprus, and also stated that this population was distinct from that of Buthus israelis (Shulov and Amitai, 1959), as follows: "Some specimens of Buthus from our region resemble specimens of the Moroccan B. o. mardochei". - "On the other hand, specimens from Cyprus, Tunisia, Libya and Somalia are different". Subsequently, the presence of a Buthus population in the island was again questioned (Gantenbein et al. 2000).

During this study, the third author (WRL) was able to find one adult female previously studied by E. Simon by the end of the $19^{\text {th }}$ century (Simon's Collection $\mathrm{N}^{\circ} 3228$ ) in the collections of the Muséum national d'Histoire naturelle, Paris. In his notes, Simon indicates that the specimen was collected in Cyprus and represented a new species, 'Buthus orientalis'. This species name, however, was never published.

Here we confirm the presence of a Buthus population in Cyprus, and a new species belonging to the "Buthus occitanus" complex is described. This new Buthus population is certainly endemic to Cyprus.

\section{Materials and methods}

Illustrations and measurements were made with the aid of a Wild M5 stereo-microscope with a drawing tube (camera lucida) and an ocular micrometer. Measurements follow Stahnke (1970) and are given in mm. Trichobothrial notations follow Vachon (1974), and morphological terminology mostly follows Vachon (1952) and Hjelle (1990). Specimens were photographed using a Nikon d100 (lens AF micro-NIKKOR $60 \mathrm{~mm} \mathrm{f} / 2.8 \mathrm{D}$ ). Digital images were edited with the assistance of Photoshop CS3 software.

\section{Abbreviations}

MNHN Museum National d'Histoire Naturelle, Paris, France.

MTAS Museum of Turkish Arachnology Society, Ankara, Turkey. 


\section{Results}

\section{Description of the new species}

\section{Buthus kunti sp. n.}

urn:lsid:zoobank.org:act:96DA8302-0891-4EF8-8D5B-DA8275325908

http://species-id.net/wiki/Buthus_kunti

Figs $1-12$

Type material: Cyprus, 1 female holotype, Karpaz Region, Dipkarpaz Town (İskele), 2 km south east, $35^{\circ} 35^{\prime} 05^{\prime \prime}$ N, 34²5'23” E, leg. H. Koç (MTAS). Paratypes: 1 subadult

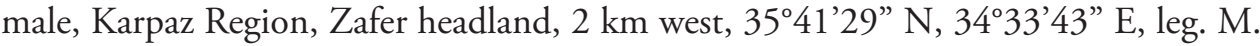
Z. Yıldız and B. Göçmen (MTAS). 1 subadult male, Güzelyurt District (Morphou), about $5 \mathrm{~km}$ south east of Güzelyurt town, leg. H. Koç (MNHN) (Fig. 13).

Note: Although Simon's female specimen may belong to the new species, we decided not to include it among the type material because (i) it is poorly preserved (ii) the precise collecting site is unknown.

Derivatio nominis: The species is dedicated to Kadir Boğaç Kunt who is the founder of the Turkish Arachnological Society.

Diagnosis: Scorpion of medium to large size, reaching a total length of $73 \mathrm{~mm}$. General coloration yellow to pale yellow, with brownish spots on the carinae of carapace; legs with diffused brownish spots. Carinae moderately to strongly marked; granulations moderately to weakly marked. Fixed and movable fingers with 12 rows of granules. Pectines with 27 to 29 teeth in males, 24-25 in female.

Relationships: Buthus kunti sp. n., belongs to the "Buthus occitanus" species complex. It can be distinguished from the other species of Buthus and in particular from $B$. israelis Shulov \& Amitai, 1959, a species distributed in the nearby region of the Middle East, by the following characters: (i) B. israelis is smaller, measuring up to $62 \mathrm{~mm}$ in total length for females; (ii) according to Levy and Amitai (1980) pectinal teeth 28-33 in males, 22-28 in females, the new species has a slightly reduced number of pectinal teeth; (iii) metasomal segment II is longer than wide in the female of the new species, whereas it is wider than long in the female of $B$. israelis; (iv) pedipalp segments are oligotrichous (sense Vachon 1952) in the new species, whereas they are polytrichous in B. israelis.

Taxonomic note: As already exposed in a recent paper (Lourenço et al. 2010), the Israeli and Sinai populations were originally described only as a variety: Buthus occitanus mardochei var. israelis Shulov \& Amitai, 1959. Subsequently, this form was raised to subspecies level as Buthus occitanus israelis (Levy and Amitai 1980). This decision followed the previous taxonomic position adopted by Vachon (1952), who considered almost all Buthus populations from North Africa and Middle East as subspecies of Buthus occitanus. However, a revision of the genus Buthus (Lourenço 2003) revealed that the species Buthus occitanus is limited to France and Spain. Most of the populations of Buthus, previously defined as subspecies and even varieties, were raised to the species level, or described as new species. In the case of Buthus occitanus israelis, it seemed that 
this population could no longer be considered as a subspecies of B. occitanus, both for morphological and especially geographical reasons. Consequently, it was raised to species level, as B. israelis (Lourenço et al. 2010). Kovař́lk (2006) examined material from Egypt and Israel and synonimized Buthus occitanus mardochei var. israelis Shulov \& Amitai, 1959 and Buthus occitanus israelis with B. intumescens. But Lourenço et al. (2010) didn't follow this synonimization and accept Buthus occitanus israelis as valid and elevated to species range.

Description based on female holotype: Measurements in Table 1. Coloration basically yellowish to pale yellow (Figures 1-3). Prosoma: carapace yellowish; carinae and eyes marked by dark pigment (Figs 1-2).

Mesosoma yellowish with carinae also marked by dark pigment, but less conspicuous than carapace. Metasomal segments yellowish; vesicle yellowish; aculeus yellowish at its base and dark reddish at its extremity. Venter yellowish; pectines pale yellow.

Table I. Morphometric values (in mm) of the female holotype of Buthus kunti sp. n.

\begin{tabular}{|c|c|}
\hline Total length & 73.3 \\
\hline \multicolumn{2}{|l|}{ Carapace: } \\
\hline - length & 8.2 \\
\hline - anterior width & 5.8 \\
\hline - posterior width & 9.4 \\
\hline Mesosoma length: & 21.4 \\
\hline \multicolumn{2}{|l|}{ Metasomal segment I: } \\
\hline - length & 5.5 \\
\hline - width & 5.7 \\
\hline \multicolumn{2}{|l|}{ Metasomal segment II: } \\
\hline - length & 6.6 \\
\hline - width & 5.7 \\
\hline \multicolumn{2}{|l|}{ Metasomal segment V: } \\
\hline - length & 8.7 \\
\hline - width & 4.8 \\
\hline - depth & 3.7 \\
\hline \multicolumn{2}{|l|}{ Telson: } \\
\hline - length & 8.0 \\
\hline - width & 4.0 \\
\hline - depth & 3.5 \\
\hline \multicolumn{2}{|l|}{ Pedipalp: } \\
\hline - Femur length & 6.6 \\
\hline - Femur width & 2.2 \\
\hline - Patella length & 7.6 \\
\hline - Patella width & 3.1 \\
\hline - Chela length & 13.2 \\
\hline - Chela width & 3.4 \\
\hline - Chela depth & 3.6 \\
\hline Movable finger: length & 8.9 \\
\hline
\end{tabular}


Chelicerae yellowish with vestigial variegated spots; fingers yellowish with dark reddish to blackish teeth. Pedipalps yellowish; fingers with dark oblique rows of denticles. Legs pale yellow with diffuse brownish spots.

Morphology: Carapace moderately to strongly granular; anterior margin almost straight and without a median concavity. Carinae strong; anterior median, central median and posterior median carinae strongly granular, with 'lyre' configuration. All furrows moderate to strong. Median ocular tubercle at the centre of carapace. Eyes separated by almost three ocular diameters (one median eye absent on the holotype). Three pairs of lateral eves of moderate size (Fig. 1). Sternum triangular, wider than

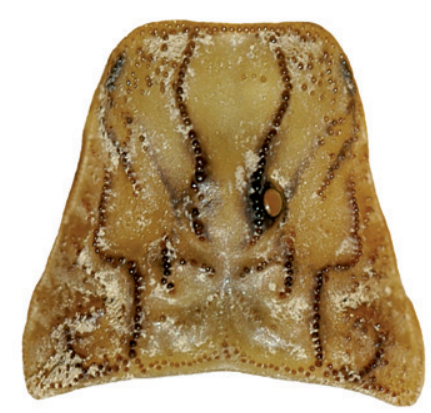

\section{1}

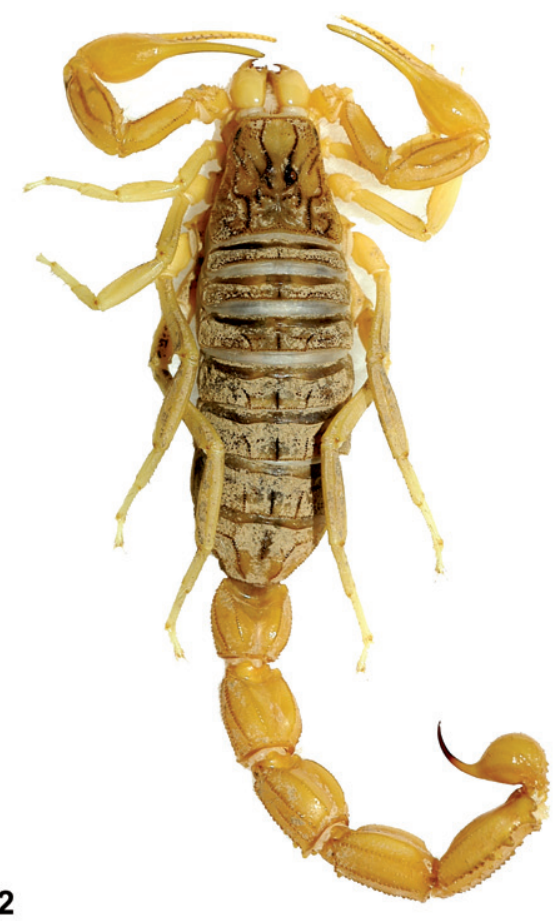

3

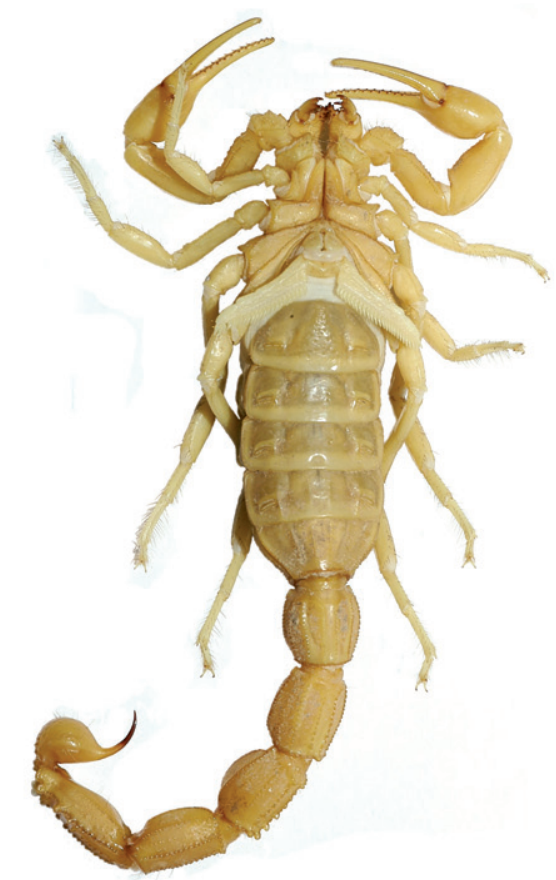

Figures I-3. Buthus kunti sp. n. I Carapace of female holotype $\mathbf{2}$ female holotype from Karpaz $\mathbf{3}$ Ditto, ventral view. 
long. Mesosoma: tergites moderately granular. Three longitudinal carinae moderately crenulate in all tergites; lateral carinae reduced in tergites I and II. Tergite VII pentacarinate. Venter: genital operculum divided longitudinally, which plate with a semitriangular shape. Pectines: pectinal tooth count: 25-24 in female holotype (28-27, 29-29 in male paratypes); middle basal lamella of the pectines not dilated. Sternites without granules, smooth with elongated spiracles; four carinae on sternite VII; other sternites acarinated and with two vestigial furrows. Metasomal segments I to III with ten crenulated carinae, ventral strongly marked on II-III with lobate granules; segment IV with eight carinae, crenulated; the first four segments with a smooth dorsal depression; segment $\mathrm{V}$ with five carinae; the latero-ventral carinae crenulate with 2-3 lobate denticles posteriorly (Fig. 5); ventral median carina not divided posteriorly; anal arc composed of 5-6 ventral teeth, and two lateral lobes. Intercarinal spaces weakly granular. Telson almost smooth; aculeus curved and only slightly shorter than the vesicle, without a subaculear tubercle (Fig. 5). Cheliceral dentition as defined by Vachon (1963) for the family Buthidae; external distal and internal distal teeth approximately the same length; basal teeth on movable finger small but not fused (Fig. 7); ventral aspect of both fingers and manus covered with long dense setae. Pedipalps: Femur pentacarinate; patella with eight carinae; all faces weakly granular; chela smooth, without carinae. Fixed and movable fingers with 12 oblique rows of granules. Internal and external accessory granules present, strong; three accessory granules on the distal end

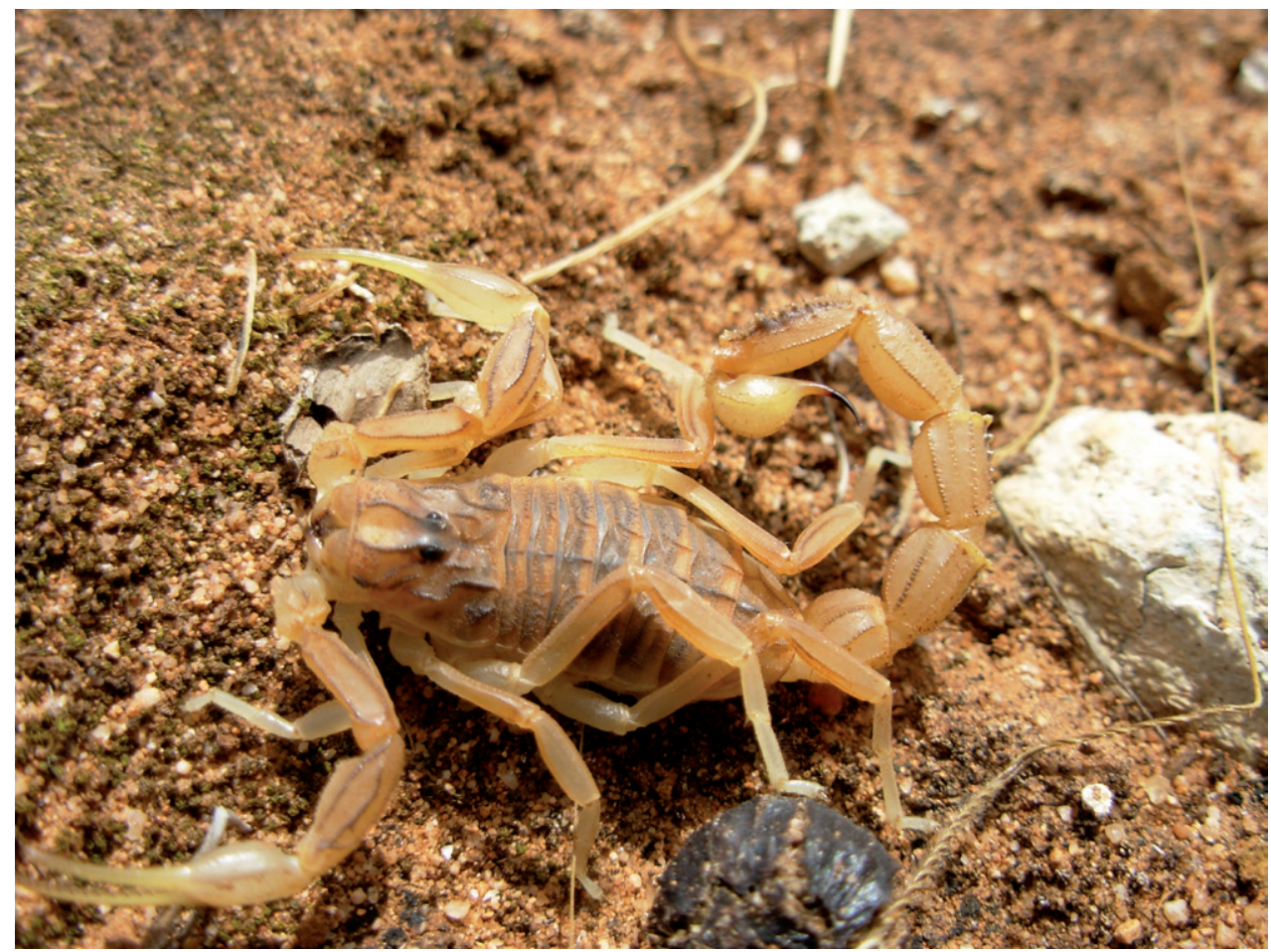

Figure 4. Buthus kunti sp. n., subadult male paratype from Zafer headland. 


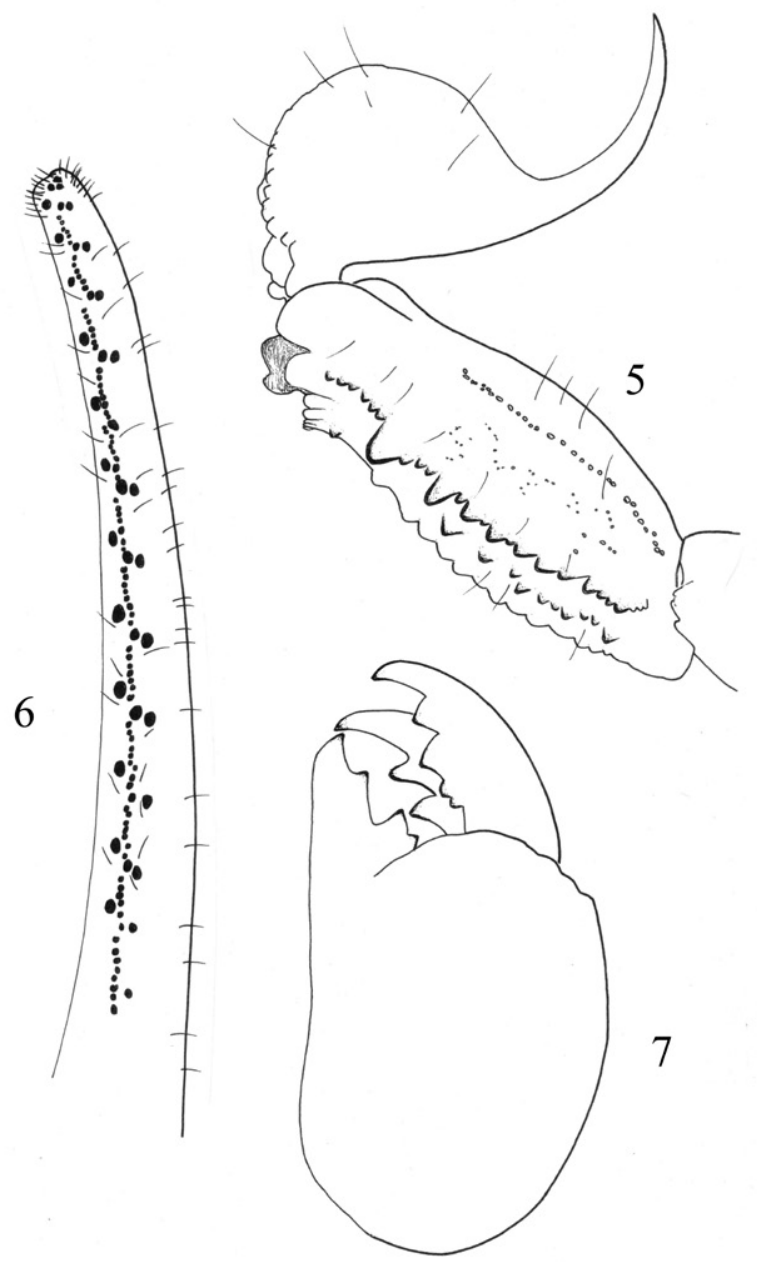

Figures 5-7. Buthus kunti sp. n. Female holotype 5 Metasomal segments V and telson, lateral aspect 6 Movable finger of pedipalp chela with rows of granules $\mathbf{7}$ Chelicera, dorsal aspect.

of the movable finger next to the terminal denticles (Fig. 6). Legs: Tarsus with two longitudinal rows of thin and long setae ventrally; tibial spur strong on legs III and IV; pedal spurs moderate on legs I to IV. Trichobothriotaxy: trichobothrial pattern of Type A, orthobothriotaxic as defined by Vachon (1974). Dorsal trichobothria of femur arranged in $\beta$-configuration (Vachon 1975) (Figs 8-12).

Ecological notes and biogeography: Cyprus Island exhibits the Mediterranean climate which is warm and rainy in winter and hot and dry in summer. Rainy season is rare and only occurs in winter in plain areas (İlseven et al. 2006). Sandy soil exists at Zafer headland locality, where the vegetation is composed of Pancratium maritimum, Cakile maritima, Limonium albidum and Pistacia lentiscus (Fig. 14). Redzina soil is present at Güzelyurt, where the habitat was steppe vegetation with small bushes. Buthus kunti sp. n. has allopatric distribution with another species endemic to Cyprus, Mesobu- 


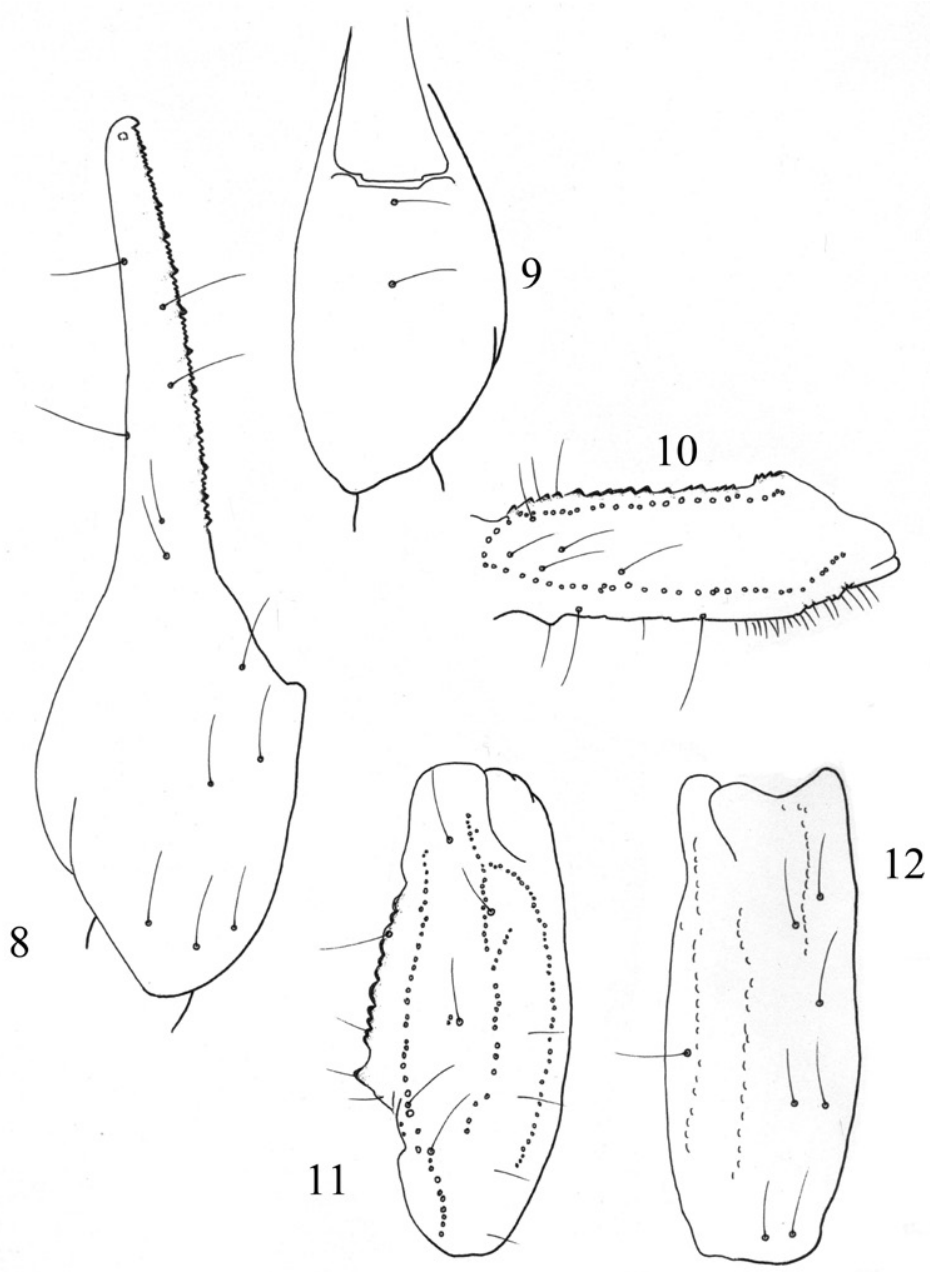

Figures 8-12. Trichobothrial pattern of Buthus kunti sp. n., female holotype. 8-9 Chela, dorso-external and ventral aspects $\mathbf{1 0}$ Femur, dorsal aspect I I-I 2 Patella, dorsal and external aspects.

thus cyprius Gantenbein \& Kropf, 2000. Interestingly, Cyprus Island is the only territory where representatives of Buthus and Mesobuthus genera have been found together.

The geological evolution of the eastern Mediterranean region, has run a series of prominent geological movements, together with the world wide sea levels rising and falling accompanying the continental glaciations leading to join and split of Cyprus and Anatolia (Robertson 1998). It is thus clear that no consensus yet as to the geological history of Cyprus; Schmidt (1960) express Cyprus was part of a united landmass of the mainland and then was broken piece of the mainland, but according to the modern geological history of the eastern Mediterranean region, Cyprus became due to tectonic movements occurring in the area, Gass (1987) supports during Mesozoic time Mt. Troodos is originated a submarine volcano that arise an oceanic island which occured at Cretaceous-Palaeocene. Whereas Kyrenia Mts (which include Pentadactylos Mt.) may- 


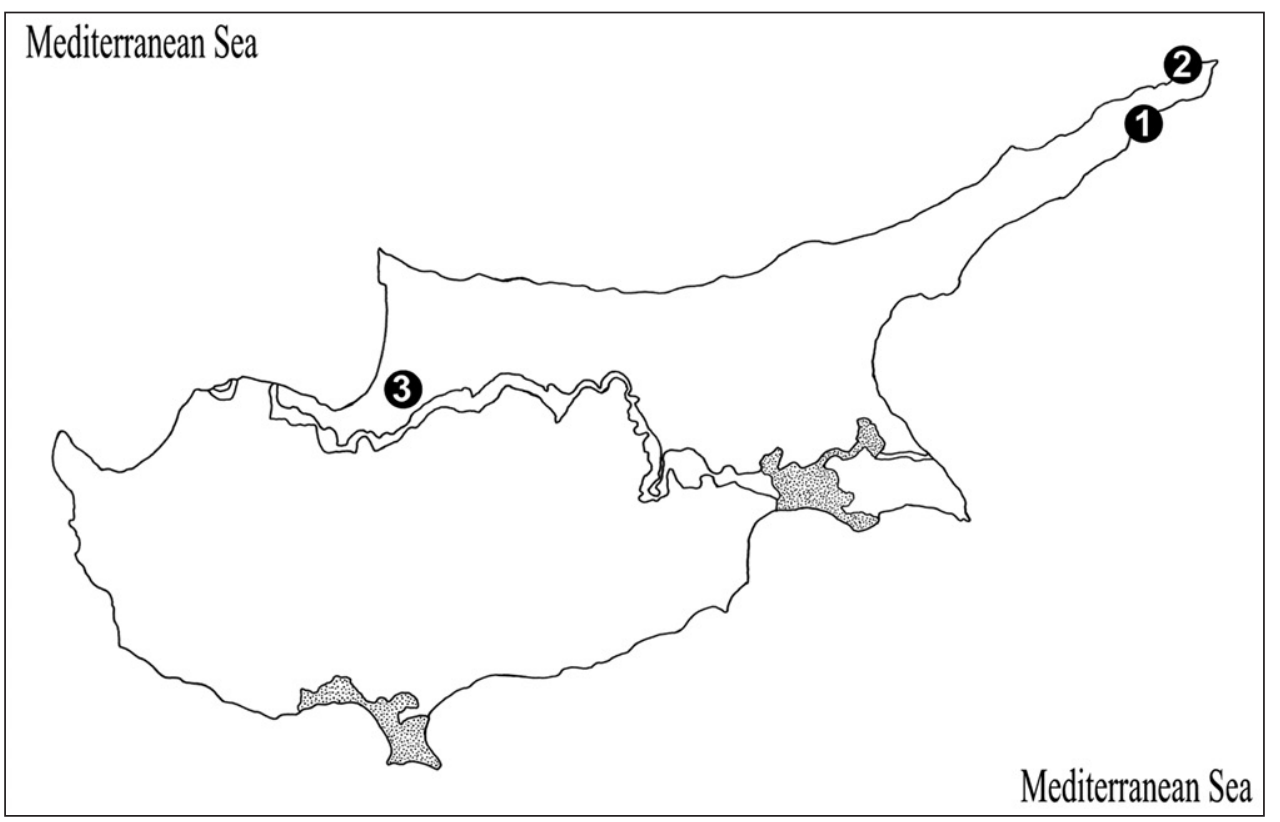

Figure 13. Map of Cyprus, showing the site where the new species was collected. I Collecting locality of holotype, Karpaz Region, Dipkarpaz Town (İskele) 2 Collecting locality of paratype, Karpaz Region, Zafer headland 3 Ditto, Güzelyurt District (Morphou).

be as a second island or as a part of the southern Taurus Mts range originated in Eocene then later separated from each other to the south (Cavazza and Wezel 2003). According to widely accepted theory is Mediterranean salinity crisis that the Mediterranean sea dried out and these two island or the Trodos island and southern Tauruian-Kyrenian peninsula connected via landbridges about 5.6 Myrs (Hsü et al. 1977; Cavazza and Wezel 2003). When the refilling of the Mediterranean basin, Cyprus terrestrial animals was isolated for around 5.2 - 5.3 Myrs (Robertson 1998; Gantenbein and Keightley 2004 ). This isolation played a major role in forming actual scorpion fauna of Cyprus and molecular and morphological phylogenetic analysis has revealed that populations of the island of Cyprus represent a divergent lineage; so these have been assigned to the species rank (i.e., Mesobuthus cyprius Gantenbein and Kropf, 2000). On the other hand, the other discussions about endemism of some snake species occurring in the two island origin of Cyprus (Troodos and Kyrenia island); Hierophis cypriensis, in only southern Cyprus (i.e., Throodos island) while Platyceps najadum (non-endemic) and Natrix tessellata (non-endemic) is distributed only in northern Cyprus (i.e., Kyrenia island) and also on the mainland (Göçmen et al. 2009). Gantenbein and Keightley (2004) stated his analyses shows that Mesobuthus cyprius occurring in Cyprus is autochthonous. Mesobuthus cyprius recorded in both southern and northern Cyprus. While Mesobuthus cyprius recorded at high elevation in Cyprus, Buthus kunti sp. n. collected at low altitude in dry condition. It is not yet clear if the distribution of new species restricted to Kyrenia island (northern Cyprus). However, Mt. Troodos run vertically and Kyrenia Mts. lay horizon- 


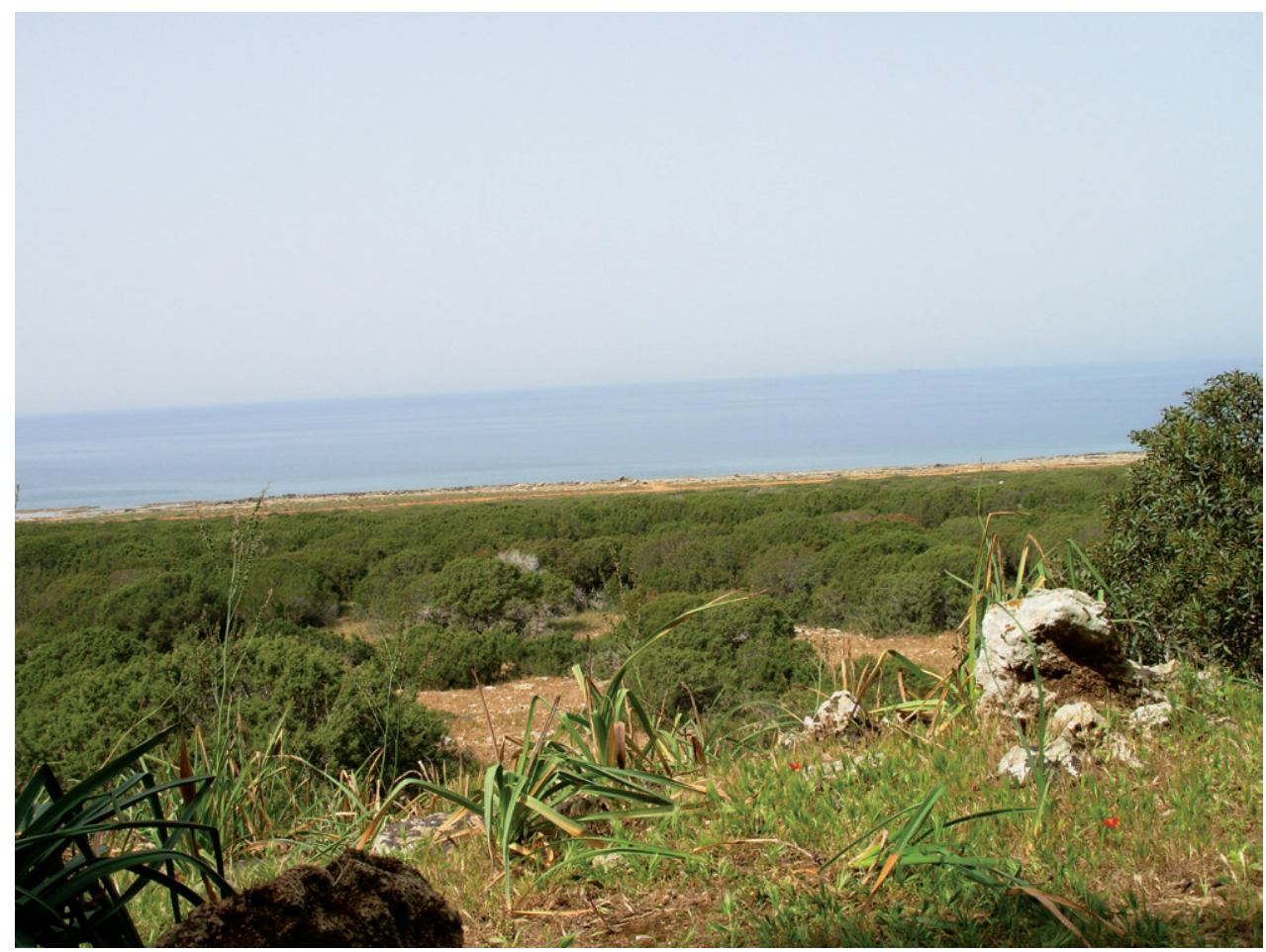

Figure 14. Buthus kunti sp. n. Habitat from Zafer headland (Sandy soil habitat).

tally with less high in Cyprus, are not usually a zoogeographic barrier there. When we take in consideration for this situation we expect the distribution of new species is all over Cyprus. Another point of view explains that as a result of the geological process, it is a localized endemic species in Kyrenia island (Pentadactylos Mt.).

Since the second record of scorpion species, a museum material, Simon's material the precise collecting site is unknown and poorly preserved, no other species have been seen in several recent field works, so the species might be very rare on the island, and should be investigated again for male specimens under suitable seasonal conditions.

Unplanned urban settlement destroys the habitats of these endemic species. Government agencies are required to take precautions to not destroy habitats.

\section{Acknowledgements}

We are most grateful to Dr. Victor Fet, Marshal University, Huntington, West Virginia, USA for useful comments to the manuscript. The first two authors wish to thank Dr. Bayram Göçmen and Mehmet Zülfü Yıldız (Ege University, Turkey) for providing specimens, photographs and literature. 


\section{References}

Cavazza W, Wezel FC (2003) The Mediterranean region - a geological primer. Episodes 26: $160-168$.

Gantenbein B, Kropf C, Largiadèr CR, Scholl A (2000) Molecular and morphological evidence for the presence of a new buthid taxon (Scorpiones: Buthidae) on the Island of Cyprus. Rev. Suisse Zool. 107: 213-232.

Gantenbein B, Keightley PD (2004) Rates of molecular evolution in nuclear genes of east mediterranean scorpions. Evolution 58: 2486-2497.

Gass IG (1987) Ophiolite: Ozeankruste an Land . In: Giesse PP (Ed) Ozeane und Kontinente, Spektrum der Wissenschaft, Heidelberg, Germany, 172-181.

Göçmen B, Kasot N, Yıldız MZ, Sas I, Akman B, Yalçınkaya D, Gücel S (2008) Results of the herpetological trips to Northern Cyprus. North-West J. Zool. 4: 139-149.

Göçmen B, Atatür MK, Budak A, Bahar H, Yıldız MZ, Alpagut-Keskin N (2009) Taxonomic notes on the snakes of Northern Cyprus, with observations on their morphologies and ecologies. Animal Biology 59: 1-30. doi:10.1163/157075609X417062

Hjelle JT (1990) Anatomy and morphology. In: Polis GA (Ed) The Biology of Scorpions, Stanford University Press, Stanford, 9-63.

Hsü KJ, Montadert L, Bernoulli D, Cita MB, Erickson A, Garrison RE, Kidd RB, Mèlierés F, Müller C, Wright R (1977) History of the Mediterranean salinity crisis. Nature 267: 399-403. doi:10.1038/267399a0

İlseven S, Hıdırer G, Tümer A (2006) Kıbrıs Coğrafyası (Geography of Cyprus), K.T. Eğitim Vakfi, Lefkoşa.

Kovařik F (2006) Review of Tunisian species of the genus Buthus with descriptions of two new species and a discussion of Ehrenberg's types (Scorpiones: Buthidae). Euscorpius 34: 1-16.

Kraepelin K (1891) Revision der Skorpione. I. Die Familie der Androctonidae. Jahrbuch der Hamburgischen wissenschaftlichen Anstalten 8: 1-144.

Levy G, Amitai P (1980) Fauna Palaestina, Arachnida I: Scorpiones, Israel Academy of Sciences and Humanities, Jerusalem, 130pp.

Lourenço WR (2002) Considérations sur les modèles de distribution et différentiation du genre Buthus Leach, 1815, avec la description d'une nouvelle espèce des montagnes du Tassili des Ajjer, Algérie (Scorpiones, Buthidae). Biogeographica 78: 109-127.

Lourenço WR (2003) Compléments à la faune de scorpions (Arachnida) de l'Afrique du Nord, avec des considérations sur le genre Buthus Leach, 1815. Rev. Suisse Zool. 110: 875-912.

Lourenço WR, Yağmur EA, Duhem B (2010) A new species of Buthus Leach, 1815 from Jordan (Scorpiones, Buthidae). Zoology in the Middle East 49: 95-99.

Robertson AHF (1998) Mesozoic-Tertiary tectonic evolution of the easternmost Mediterranean area: integration of marine and land evidence. In: Robertson AHF, Emeis KC, Richter C, Camerlenghi A (Eds) Proceedings of the Ocean Drilling Program, Scientific Results, Vol. 160. Collage Station, TX (Ocean Drilling Program), 723-782.

Schmidt WE (1960) Zur Struktur und Tektonik der Insel Cypern. Geologische Rundschau 50: 375-395. doi:10.1007/BF01786852 
Stahnke HL (1970) Scorpion nomenclature and mensuration. Entomol News 81: 297-316.

Vachon M (1952) Etudes sur les scorpions. Publications de l'Institut Pasteur d'Algérie, Algeria, 782pp.

Vachon M (1963) De l'utilité, en systématique, d'une nomenclature des dents des chélicères chez les Scorpions. Bulletin du Muséum national d'Histoire naturelle 35: 161-166.

Vachon M (1974) Etude des caractères utilisés pour classer les familles et les genres de Scorpions (Arachnides). 1. La trichobothriotaxie en arachnologie. Sigles trichobothriaux et types de trichobothriotaxie chez les Scorpions. Bulletin du Muséum national d'Histoire naturelle 104: 857-958.

Vachon M (1975) Sur l'utilisation de la trichobothriotaxie du bras des pédipalpes des Scorpions (Arachnides) dans le classement des genres de la famille des Buthidae Simon. Comptes Rendus des Séances de l'Académie de Sciences 281: 1597-1599. 